\section{BRAZIULIAN JOURNAL}

OF MEDICAL AND BIOLOGICAL RESFARCH

www.bjournal.com.br
ISSN 0100-879X

Volume 43 (02) 124-225 February 2010

BIOMEDICAL SCIENCES

AND

CLINICAL INVESTIGATION

Braz J Med Biol Res, February 2010, Volume 43(2) 176-185

Rat DIx5 is expressed in the subventricular zone and promotes neuronal differentiation

H.F. Shu, F.Y. Gao, C.Q. Zhang, S.Y. Liu, Z.Y. Zhang, Y.C. Song, K.J. Qiu and H. Yang

The Brazilian Journal of Medical and Biological Research is partially financed by
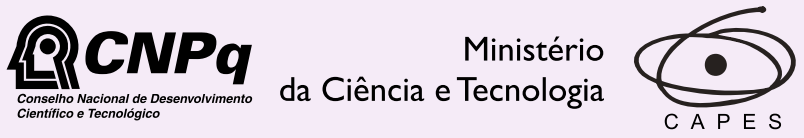

Ministério da Educação

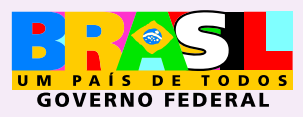

Institutional Sponsors 


\title{
Rat Dlx5 is expressed in the subventricular zone and promotes neuronal differentiation
}

\author{
H.F. Shu*, F.Y. Gao*,a, C.Q. Zhang, S.Y. Liu, Z.Y. Zhang, \\ Y.C. Song, K.J. Qiu and H. Yang \\ Department of Neurosurgery, Xinqiao Hospital, Third Military Medical University, Chongqing, China
}

\begin{abstract}
The molecular mechanisms and potential clinical applications of neural precursor cells have recently been the subject of intensive study. Dlx5, a homeobox transcription factor related to the distal-less gene in Drosophila, was shown to play an important role during forebrain development. The subventricular zone (SVZ) in the adult brain harbors the largest abundance of neural precursors. The anterior SVZ (SVZa) contains the most representative neural precursors in the SVZ. Further research is necessary to elucidate how DIx5-related genes regulate the differentiation of SVZa neural precursors. Here, we employed immunohistochemistry and molecular biology techniques to study the expression of DIx5 and related homeobox genes Er81 and Islet1 in neonatal rat brain and in in vitro cultured SVZa neural precursors. Our results show that DIx5 and Er81 are also highly expressed in the SVZa, rostral migratory stream, and olfactory bulb. Islet1 is only expressed in the striatum. In cultured SVZa neural precursors, DIx5 mRNA expression gradually decreased with subsequent cell passages and was completely lost by passage four. We also transfected a DIx5 recombinant plasmid and found that DIx5 overexpression promoted neuronal differentiation of in vitro cultured SVZa neural precursors. Taken together, our data suggest that Dlx5 plays an important role during neuronal differentiation.
\end{abstract}

Key words: Neural precursors; Dlx5; Green fluorescent protein; Recombinant plasmids

\section{Introduction}

Neural precursors are a population of self-renewing cells with the potential to differentiate into multiple lineages. Under appropriate conditions, these cells can undergo asymmetric cell division, giving rise to neurons and neuronal precursors that continue to self-renew (for a review, see Ref. 1). Therefore, these neural precursors can be used to replace injured neurons. However, the molecular mechanisms underlying neural precursor differentiation are unknown, limiting the clinical utilization of neural precursors. This research area is currently under intensive investigation. Elucidating the molecular mechanisms of neural precursor differentiation will greatly facilitate the clinical application of neural precursors to the treatment of central nervous system damage.

Populations of neural precursors are present in numerous areas of embryonic and adult mouse brains, including the midbrain, hippocampus, subventricular zone (SVZ), and ganglionic eminence. Neural precursors are found in the
SVZ of human embryos and adults, but are concentrated in the lateral ventricles of the SVZ during adulthood $(2,3)$. The anterior SVZ (SVZa) contains most of the neural precursors in the SVZ. Starting from the embryonic stage and continuing under the regulation of dorsoventral signaling, SVZa precursors migrate toward the core of the olfactory bulb (OB) via a highly specific pathway, the rostral migratory stream (RMS). The precursors from the central area then scatter toward the granule and peripheral layers, where they differentiate into mature OB interneurons (4). This process of directional migration, proliferation, and differentiation provides continual renewal of OB interneurons $(5,6)$. This renewal is apparent in the neonatal stage and persists in adults (7). Therefore, SVZa neural precursors are an ideal model for the study of neuronal precursor migration and differentiation (8).

A multitude of molecules have been shown to regulate the migration and differentiation of SVZa neural precursors.

Correspondence: H. Yang, Department of Neurosurgery, Xinqiao Hospital, Third Military Medical University,

183 Xinqiao Main Street, Shapingba District, Chongqing 400037, China. Fax: +86-23-6521-8204. E-mail: huiyang64@yahoo.com

${ }^{*}$ These authors contributed equally to this study.

aThe present address of F.Y. Gao is Department of Neurosurgery, Guizhou Provincial People's Hospital, China. 
Our previous studies have indicated that Pax6, BMPs, Wnt1 , and Mash1 play important roles in these processes (9). Recently, Dlx5 was shown to be involved in the development of the central nervous system, especially the forebrain (10). Distal-less homeobox (Dlx) genes belong to a family of highly conserved homeobox transcriptional factors homologous to the Drosophila distal gene (for a review, see Ref. 11) and are located on chromosomes in a paired manner. Three pairs have been cloned so far, Dlx1 and 2, Dlx3 and 4, and DIx5 and 6, all of which are important during forebrain development (for reviews, see Refs. 11 and 12). Among these, DIx5 is crucially involved in the development of OB neurons. Studies using knock-out mice have shown that DIx5 plays a critical role in the differentiation of $O B$ interneurons and the axonal contact of $\mathrm{OB}$ receptor neurons (13). DIX5 is also required for the maturation of SVZa precursors in the OB (14). Furthermore, it specifically regulates differentiation of the precursors related to olfactory system development and is therefore essential for neural development after birth (10). It has been hypothesized that DIx5 may be involved in the migration and differentiation of SVZa precursors. Further studies are required to address the function and molecular mechanisms of DIx5.

Unfortunately, given that Dlx5 knock-out is lethal, it is not feasible to study DIx5 function in the neonatal stage, which happens to be the major period for OB interneuron maturation. Therefore, we have been using multiple techniques, such as tissue culture, immunohistochemistry (IHC), recombinant plasmids, RT-PCR, and flow cytometry (FCM), to study the expression and localization of DIx5 and related homeobox proteins in the brains of neonatal rats. Furthermore, we studied the effect of DIx5 on neuronal differentiation of cultured SVZa precursors.

\section{Material and Methods}

\section{Ethics statement}

All experiments were performed with the approval of the Animal Ethics Committee of the Third Military Medical University. All experimental procedures were conducted in accordance with the National Institutes of Health "Guide for the Care and Use of Laboratory Animals" (NIH publication No. 80-23, revised in 1996). All efforts were made to minimize animal suffering and to reduce the number of animals used.

\section{Cell culture}

According to the procedures described by Liu et al. (9) and Luskin et al. (15), neonatal Wistar rats (P0) were anesthetized with $0.3 \%$ pentobarbital and the SVZa and dorsolateral striatum brain areas were dissected out. We prepared the single cell suspension using mechanical dissociation. After counting living cells with Trypan blue, $1 \times 10^{6}$ cells were seeded into culture flasks and cultured in serum-free medium (DMEM/F12, 1:1) supplemented with B27 (2\%, Gibco, USA) and bFGF (20 ng/mL, Sigma, USA). After the formation of primary clones, a single cell suspension was prepared by mechanical dissociation of these primary clones and $1 \times 10^{6}$ cells were again seeded into culture flasks. Cell passage was performed by mechanical dissociation every 3-4 days.

\section{Plasmid construction and transfection}

The mouse Dlx5 (mDlx5) fragment was removed from pBluescriptBSK-mDIx5 (a gift from Prof. Giovanni Levi) by EcoRI and Kpnl double digestion. The mDIx5 fragment was then ligated into a pEGFP eukaryotic expression vector (Clontech, Canada) to yield pEGFP-mDIx5, which encodes a fusion protein containing enhanced green fluorescent protein (EGFP) and DIx5.

SVZa neural precursors $\left(5 \times 10^{6}\right)$ from the fourth passage were transfected with $5 \mu \mathrm{g}$ of the pEGFP-mDIx 5 plasmid using Nucleofector ${ }^{\mathrm{TM}}$ (Amaxa, USA). Some cells were transfected with the pEGFP plasmid without $\mathrm{mDIx} 5$ as a negative control. Cells were then seeded onto 6 -well plates $\left(1 \times 10^{6}\right.$ cells per well) and cultured in the presence of $10 \%$ fetal bovine serum.

\section{Immunohistochemistry staining}

Cultured neural precursor cells from the third passage were treated with fetal bovine serum (FBS) for $2 \mathrm{~h}$ in order to induce differentiation before immunofluorescent staining. Cells were collected and fixed in $4 \%$ paraformaldehyde (PFA) at room temperature for $30 \mathrm{~min}$. Following washing with PBS, the cells were plated onto poly-lysine-coated slides and allowed to dry in a $37^{\circ} \mathrm{C}$ incubator. Plated cells were incubated in PBS with 5\% normal goat serum at room temperature for $30 \mathrm{~min}$. Primary antibodies against neuron-specific enolase (NSE) (1:100, Sigma), glial fibrillary acidic protein (GFAP) (1:100, Sigma), 2',3'-cyclic nucleotide 3'-phosphodiesterase (CNP; 1:100, Sigma), nestin (1:100, Santa Cruz, USA), DIx5 (1:100, Santa Cruz), Er81 (1:100, Santa Cruz), or Islet1 (1:100, Santa Cruz) were then added. Primary antibody binding was detected following incubation with fluorescently labeled secondary antibody goat anti-rabbit IgG-Cy3 (1:100 dilution, Sigma) or goat anti-rabbit IgG-FITC (1:100 dilution, Sigma) at $37^{\circ} \mathrm{C}$ for $30 \mathrm{~min}$. The slides were mounted with $50 \%$ glycerol and immediately observed under a fluorescence microscope (Leica, Germany).

To analyze the distribution of DIx5, Er81 and Islet1 proteins in the brain, neonatal rats were anesthetized with $0.3 \%$ pentobarbital and perfused with $10 \mathrm{~mL}$ saline and 50 $\mathrm{mL}$ pre-chilled $4 \%$ PFA. After perfusion, the brains were dissected out, postfixed in 4\% PFA for $24 \mathrm{~h}$, and dehydrated in $30 \%$ sucrose for $48 \mathrm{~h}$. Transverse slices $(20 \mu \mathrm{m})$ were then cut with a cryostat and collected on polylysine-coated slides. Primary antibodies against DIx5 (1:200, Santa Cruz), Er81 (1:200, Santa Cruz) and Islet1 (1:200, Santa Cruz) were used. Immunofluorescent staining of these slices was completed as described above. 


\section{RNA extraction and RT-PCR}

Total cellular RNA from the first four passages of cultured SVZ neural precursors were extracted using RNArose kits (HuaXun Inc., China). The final product of RNA was quantified by absorption and verified by agarose gel electrophoresis. The PCR primer sequences and PCR conditions for rat DIx5 and Er81 are listed in Table 1. Rat GAPDH was used as an internal standard.

\section{Flow cytometry measurement}

Differentially treated neural precursors were digested with $0.25 \%$ pancreatin, rinsed with D-Hank's solution and centrifuged three times at $250 \mathrm{~g}$ for $5 \mathrm{~min}$. NSE immunofluorescence staining was then performed. The samples were fully mixed and 3 samples from each group were used for FCM detection (type PLUS of FACstar, Becton Dickinson, USA).

\section{Statistical analysis}

Data are reported as means $\pm \mathrm{SD}$. Statistical analysis was performed by one-way ANOVA. Differences were considered to be statistically significant when $P<0.05$.

\section{Results}

\section{Culture and identification of neural precursors}

After 2 days of culture in serum-free medium, most of the primary cells isolated from the SVZa or the dorsolateral striatum died. However, some cells formed multicellular spheres and continued to proliferate. After cell passaging, the number of cells increased rapidly and the cell spheres expanded and became suspended in the medium to form neurospheres. As the cells proliferated, the number and size of neurospheres increased, and they tended to grow adherently (Figure 1A). After induction with $10 \%$ FBS for $2 \mathrm{~h}$, the

Table 1. Primer sequence and reaction conditions for PCR experiments.

\begin{tabular}{lllcc}
\hline Genes & \multicolumn{1}{c}{ Sense primer $\left(5^{\prime} \rightarrow 3^{\prime}\right)$} & Anti-sense primer $\left(5^{\prime} \rightarrow 3^{\prime}\right)$ & Renaturation temperature & Amplification length \\
\hline mDlx5 & AGAGTCCCAAGCATCCGATCC & CCAGCACAACACTGTAGTCCC & $56.8^{\circ} \mathrm{C}$ & $897 \mathrm{bp}$ \\
rDIx5 & TTATGCGGACTACGGCTACG & GGCAGGTGGGATTGATTGA & $52.3^{\circ} \mathrm{C}$ & $543 \mathrm{bp}$ \\
Er81 & AAGGGTCCCAGGCAGTTCTAT & GATGCTCTTCAGGCTCAATCA & $52.9^{\circ} \mathrm{C}$ & $229 \mathrm{bp}$ \\
GAPDH & TGCTGAGTATGTCGTGGAGT & AGTCTTCTGAGTGGCAGTGAT & $55.0^{\circ} \mathrm{C}$ & $289 \mathrm{bp}$ \\
\hline
\end{tabular}
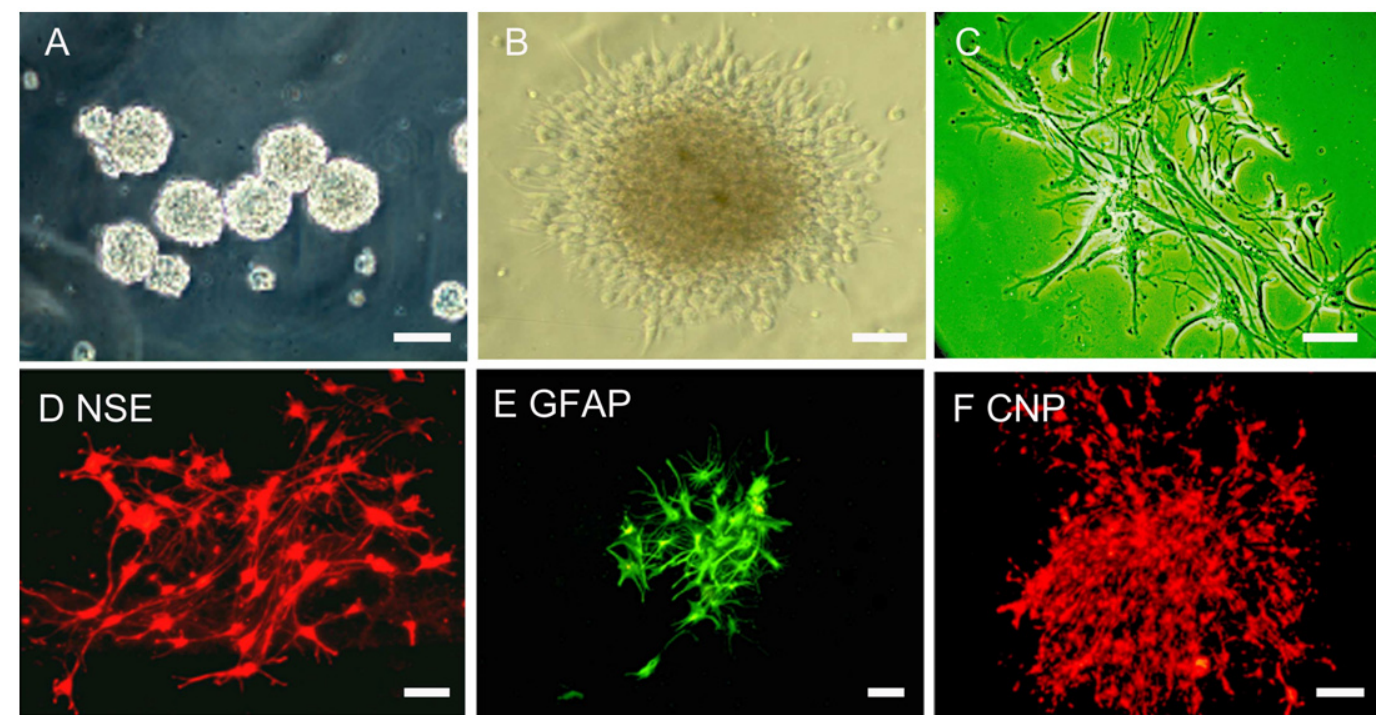

Figure 1. In vitro culture of anterior subventricular zone neural precursors. The light microscope images show that the subventricular zone neural precursors form neurospheres in suspension culture and rapidly increase in size and tend to grow together (A). After 2 $\mathrm{h}$ of induction with $10 \%$ fetal bovine serum, a light microscope image indicates that the neurosphere starts to adhere to the culture plate (B). The neurospheres shown in $C$ are at the furthermost periphery. The images of immunohistochemical staining show that the neurospheres were positive for the antibodies against NSE (D), GFAP (E), and CNP (F). NSE = neuron-specific enolase; GFAP = glial

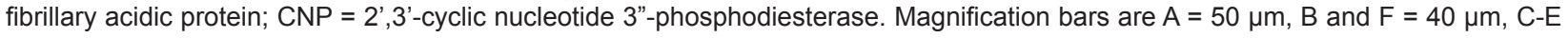
$=20 \mu \mathrm{m}$. 
neurospheres started to adhere to the plate and to differentiate (Figure 1B). The peripheral edges of the neurospheres formed short and branchless radial protrusions (Figure 1C). After two additional days of culture, these protrusions became longer and thinner, with more and more branches. Small neurospheres were almost completely differentiated. Immunostaining results confirmed the expression of GFAP, NSE, and CNP (Figure 1D-F). These results suggested that cultured neurospheres were capable of differentiating in vitro into neuronal cell types, thus highlighting a basic feature of neural precursors.

\section{Expression of DIx5, Er81 and Islet1 in neurospheres from the SVZa and dorsolateral striatum}

Immunostaining revealed that these neurospheres from the SVZa were positive for Er81, DIx5, and nestin proteins (Figure 2A-C) but negative for Islet1 expression (Figure 2D).
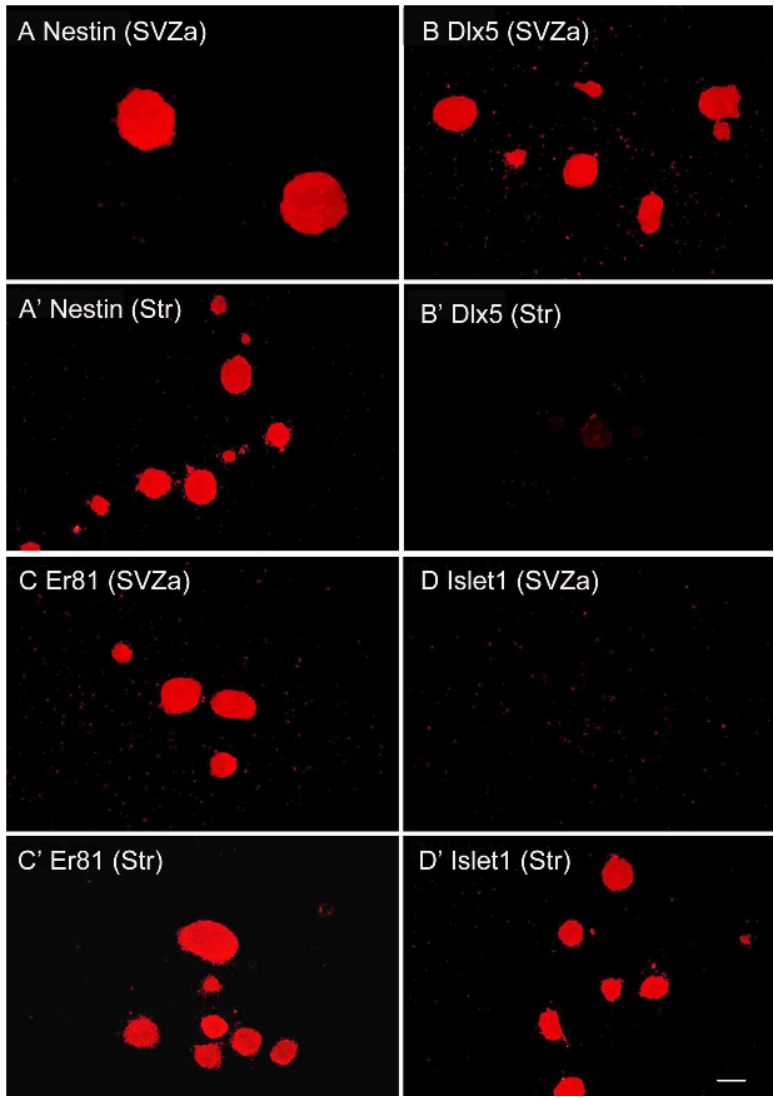

Figure 2. Expression of nestin (A, A'), Dlx5 (B, B'), Er81 (C, C'), and Islet1 ( $\left.D, D^{\prime}\right)$ in the neurospheres from anterior subventricular zone (SVZa) and striatum (Str). The neurospheres from the SVZa are positive for nestin (A), which is a precursor cell marker. They are also positive for DIx5 (B) and Er81 (C), but negative for Islet1 (D). However, the neurospheres from the striatum are positive for nestin (A'), Er81 (C') and Islet1 (D') but negative for DIx5 (B'). Magnification bar $=50 \mu \mathrm{m}$ for all panels.
However, the neurospheres from the dorsolateral striatum were positive for Er81, Islet1, and nestin proteins (Figure $\left.2 A^{\prime}, C^{\prime}, D^{\prime}\right)$ but negative for DIx5 expression (Figure 2B').

\section{Localized expression of DIx5, Er81, and Islet1 in SVZ-RMS-OB}

Stenman et al. (16) have previously analyzed the expression of DIx5/6, Er81, and Islet1 at embryonic day $\mathrm{E} 12.5, \mathrm{E} 16.5$ and in P0 mice. Similar to their reports, the $\mathrm{IHC}$ results of the present study revealed that Er81 (Figure $3 A-C, E, F)$ and DIx5 (Figure 3A'-C',E',F') were widely distributed in various brain areas of neonatal rats $(P 0)$, such as the SVZa-RMS-OB, hippocampus, and subcortex. Islet1 was not expressed in the SVZa-RMS-OB, but was expressed in both the dorsolateral and ventral striatum (Figure 3G,H). However, the expression pattern for Er81 and Dlx5 observed here was different from Stenman's results (16). We observed that Er81 was also widely expressed in both the dorsolateral and ventral striatum (Figure 3D), while Stenman et al. (16) reported that Er81 was observed in scattered striatal neurons. In addition, our data showed that DIx 5 was negatively expressed in the striatum (Figure 3C'-D'), while Stenman et al. (16) showed that Dlx proteins were positively expressed in the striatum. Thus, we think that the difference in the expression pattern of Dlx proteins in the striatum might be caused by multiple factors. For example, the Stenman study used specimens and DIx antibodies from different sources that might have contributed to the differences observed. Furthermore, the expression pattern of Dlx proteins in Stenman's study may reflect the distribution of DIX1, DIx2, DIx3, DIx4, and DIx6 in the developing brain, while in the present study it specifically reflected the SVZa-RMS-OB location of the DIx5 protein in the newborn rat brain.

\section{The effects of mDIx 5 on the morphology and differentiation of SVZa neural precursors}

Green fluorescence first appeared $4 \mathrm{~h}$ after pEGFPmDlx5 transfection and dramatically increased at $8 \mathrm{~h}$, peaking 24 to $48 \mathrm{~h}$ post-transfection and lasting several days. At $24 \mathrm{~h}$ post-transfection, total cellular RNA was extracted, and mDIx5 mRNA could be detected by RT-PCR (Figure 4A: the product is $897 \mathrm{bp}$ ). At 24 and $48 \mathrm{~h}$ posttransfection, the target $58-\mathrm{kDa}$ protein could be detected by Western blot (Figure 4B), indicating that the transfected GFP-mDIx5 fusion protein was expressed robustly. These results indicated that we could use this system to study the role of DIx5 in SVZa neural precursors further.

The transfected SVZa neural precursors were cultured for $24 \mathrm{~h}$ and then stained with NSE-Cy3. As shown in Figure 5 , the transfected cells were GFP-positive (Figure 5A) and differentiated to protrusion-bearing cells (Figure $5 B$ ). The most GFP-positive cells were positive for NSE-Cy3 (Figure $5 \mathrm{C}-\mathrm{E}$ ), indicating that the neural precursors that expressed mDIx5 successfully differentiated into neurons. 
However, not all GFP-positive cells were positive for NSE (data not shown). As a negative control experiment, some cells were transfected with an empty pEGFP plasmid that did not contain mDlx5 (Figure 5F). The NSE-staining results indicated that only a few scattered GFP-positive cells expressed NSE (Figure 5G,H).

In passages 1 through 3 of the in vitro cultured SVZa neural precursors, expression of endogenous DIx5 (rat DIx5) mRNA could be detected by RT-PCR (Figure 6, left panel). However, endogenous DIx5 could no longer be detected in
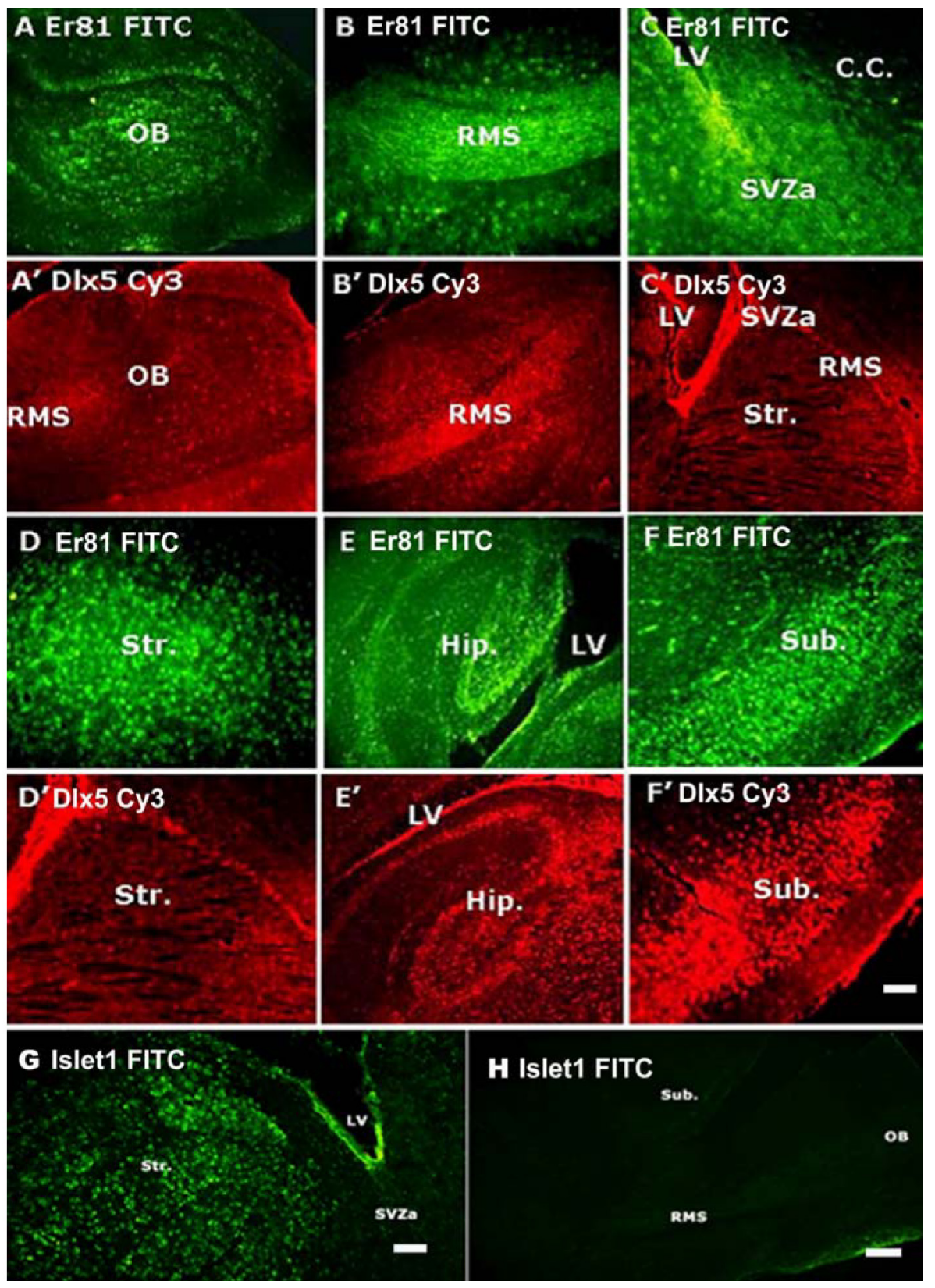

\section{H Islet1 FITC}

sub.

Figure 3. Expression of Er81 (A-F), Dlx5 ( $\left.A^{\prime}-F^{\prime}\right)$, and Islet1 (G-H) at P0. Er81 proteins are widely expressed in various brain areas including OB (A), RMS (B), SVZa (C), striatum (D), hippocampus (E), and subcortex (F). Similarly, Dlx5 are also located in the OB $\left(A^{\prime}\right)$, RMS (B'), SVZa $\left(C^{\prime}\right)$, hippocampus (E'), and subcortex (F'), but not in the striatum (C', D'). Islet1 was not expressed in the SVZa, RMS and OB but was expressed in the striatum (Figure $3 \mathrm{G}, \mathrm{H}$ ). OB = olfactory bulb; RMS = rostral migratory stream; SVZa = anterior subventricular zone; LV = lateral ventricle; C.C. = corpus callosum; Str. = striatum; Hip. = hippocampus; Sub. = subcortex. Magnification bars are $A-F$ and $A^{\prime}-F^{\prime}=50 \mu \mathrm{m}, G=30 \mu \mathrm{m}, \mathrm{H}=100 \mu \mathrm{m}$. 


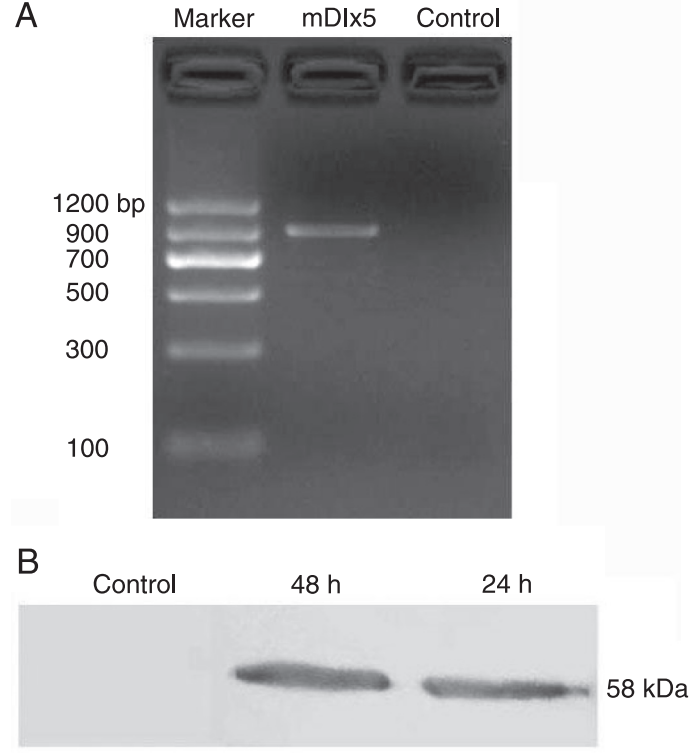

Figure 4. Expression of mouse Dlx5 (mDlx5) in anterior subventricular zone (SVZa) precursors transfected with pEGFP-mDIx5. Agarose gel electrophoresis analysis of RT-PCR showed the expression of mDlx5 RNA in SVZa precursors $24 \mathrm{~h}$ after transfection (A). Lane $M$ is the marker, the middle lane $(\mathrm{mDl} \times 5)$ is from cells transfected with pEGFP-mDIx5, and the right lane (control) is from cells without transfection of pEGFP-mDIx5. $B$, Western blot analysis showed the expression of mDlx5 proteins in SVZa precursors at 24 and $48 \mathrm{~h}$ after transfection. passage 4 precursors (Figure 6, middle panel). In contrast, Er81 mRNA was detected in different passages of SVZa precursors (Figure 6 , middle panel). When the exogenous $\mathrm{mDl} 55$ gene was transfected into passage 4 precursors, the expression of mDlx5 and of endogenous rat Dlx5 mRNA appeared after $24 \mathrm{~h}$ of culture (Figure 6, right panel).

Next, we used FCM analysis to determine the extent of neuronal differentiation in precursor populations expressing different amounts of Dlx5. As shown in Figure 7, 19.2 $\pm 0.8 \%$ of the passage 3 precursors with endogenous DIx5 mRNA could differentiate to NSE-positive neurons. In passage 4 precursors, which did not have DIx 5 mRNA, a significantly smaller fraction of cells (only $8.3 \pm 0.4 \%$; $P$ $<0.05$ ) differentiated to NSE-positive cells. Intriguingly, in the same background, the neuronal differentiation rate was enhanced to $35.7 \pm 0.6 \%$ when mDlx 5 was overexpressed. Therefore, we conclude that overexpression of DIx5 ( $P$ $<0.05)$ significantly promoted neuronal differentiation of SVZa precursors.

\section{Discussion}

In this study, we showed that the precursors from SVZa were positive for DIx5 and Er81 but negative for Islet1, while the precursors from the dorsolateral striatum were positive for Er81 and Islet1 but negative for DIx5. Similar to that reported in a previous investigation (16), the present IHC data indicated that DIx5 and Er81 are widely expressed
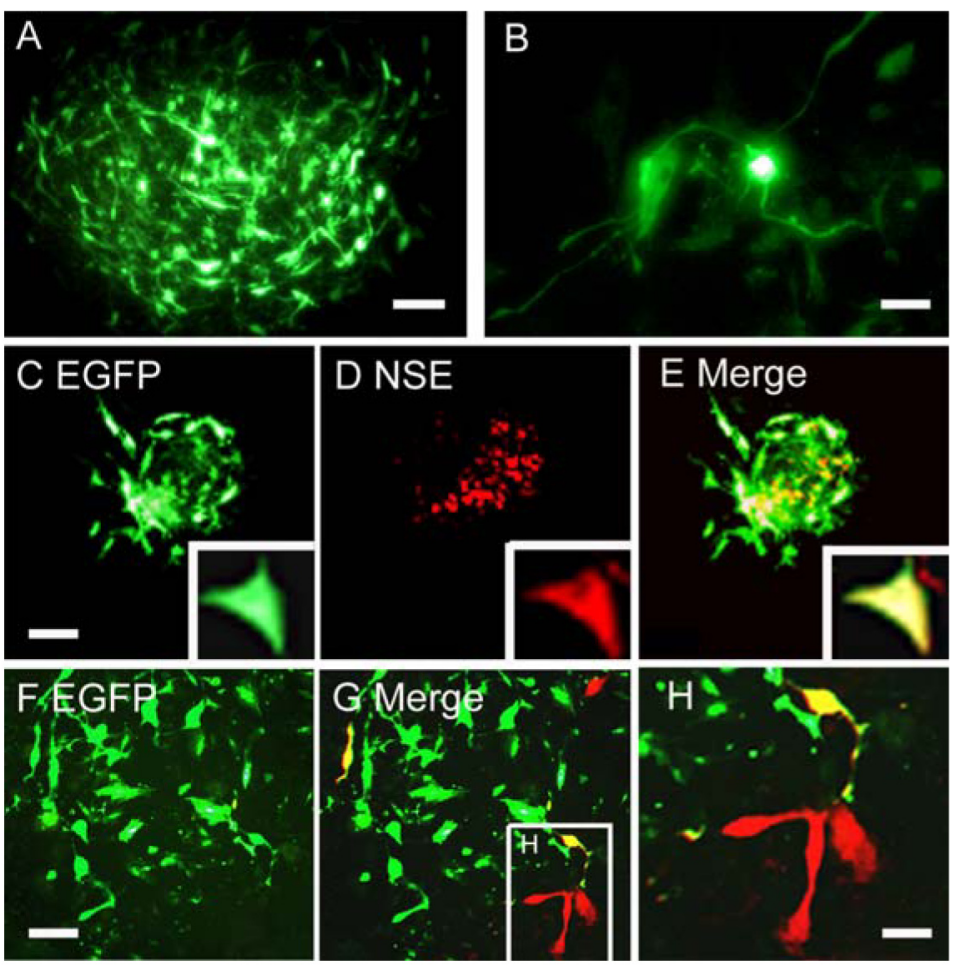

Figure 5. Expression of GFP and NSE in anterior subventricular zone (SVZa) neural precursors transfected with pEGFP-mDIx5. A, After 24 $\mathrm{h}$ of transfection with pEGFP-mDIx5, the majority of SVZa neurospheres expressed GFP. B, Representative GFP-positive SVZa neural precursors. Double-staining with GFP (C) and NSE-Cy3 (D) reveals that most of GFP-positive neurospheres/cells were differentiated into neurons $(E)$. However, cells tranfected with the pEGFP plasmid without mDIx5 $(F)$ show low rates of NSE staining $(G, H)$. GFP = green fluorescent protein; EGFP = enhanced green fluorescent protein; NSE = neuron-specific enolase. Magnification bars are $A=10 \mu \mathrm{m} ; \mathrm{B}, \mathrm{H}=30 \mu \mathrm{m} ; \mathrm{C}-\mathrm{E}$ $=100 \mu \mathrm{m} ; \mathrm{F}, \mathrm{G}=15 \mu \mathrm{m}$ 


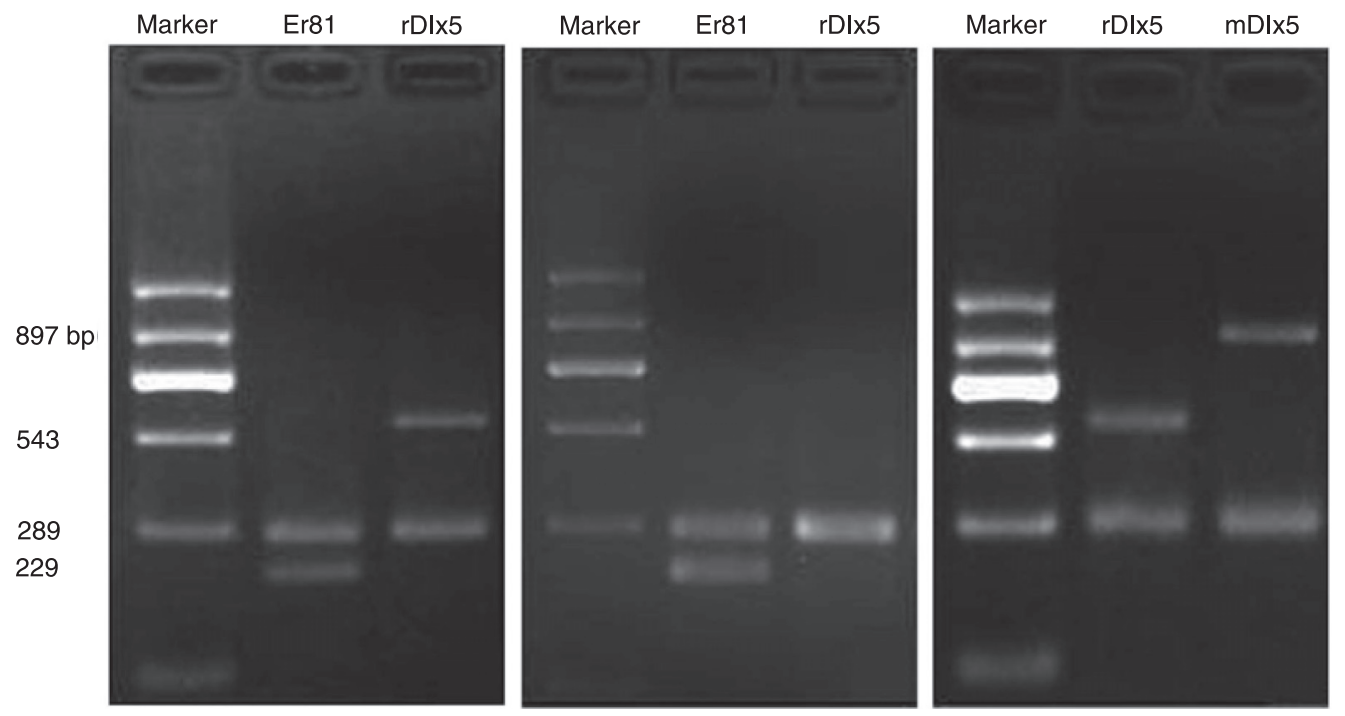

Figure 6. Expression of DIx5 and Er81 mRNA in anterior subventricular zone (SVZa) precursors. Passage 3 (left panel) and passage 4 (middle panel) of the cultured SVZa precursors were analyzed. Er81 and endogenous DIx5 (rDIx5) mRNA detection by RT-PCR followed by agarose electrophoresis analysis. The products are 229 and $543 \mathrm{bp}$, respectively. Right panel, Expression of endogenous DIx5 (rDIx5) and transfected DIx5 (mDIx5) mRNAs by RT-PCR followed by agarose gel electrophoresis analysis. After transfection with pEGFP-mDIx5, mDIx5 (897 bp) and rDIx5 expression can be detected in passage 4 precursors. Rat GAPDH was used as an internal standard; the product is $289 \mathrm{bp}$. GAPDH = glyceraldehyde 3-phosphate dehydrogenase.
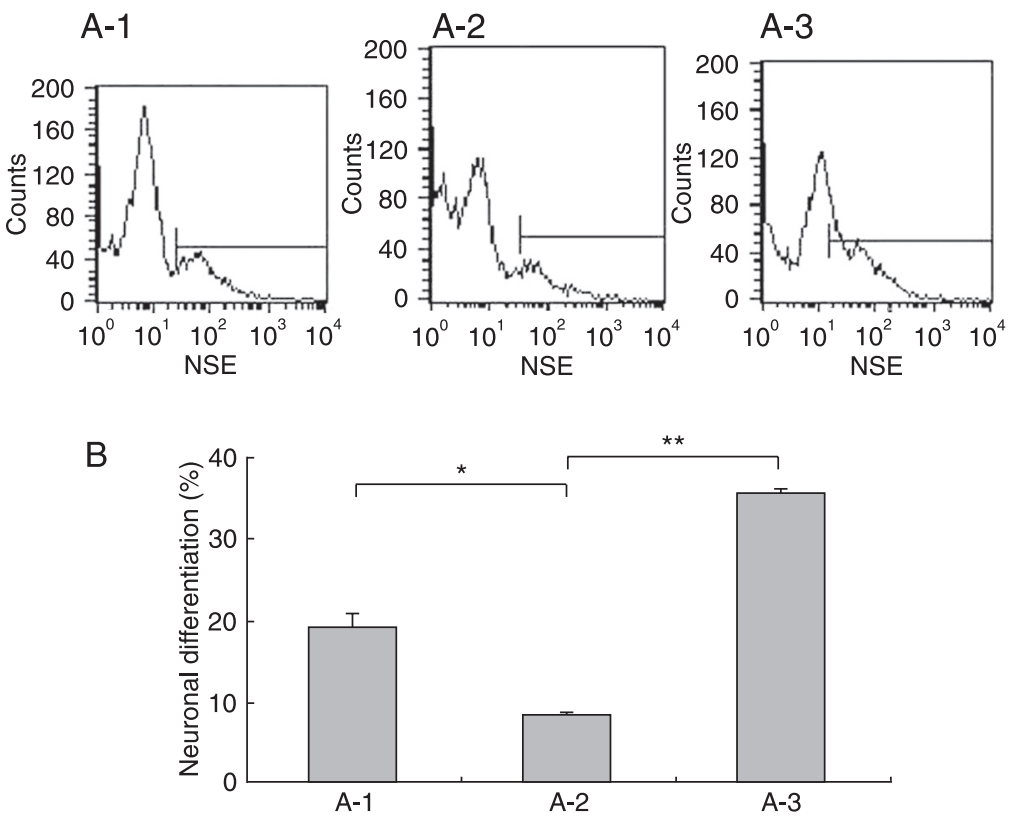

Figure 7. Determination of percent neuronal differentiation in subventricular zone (SVZ) precursors with different Dlx5 levels. $A$, Flow cytometry measurement was used to quantify NSE-positive cells after the anterior SVZ (SVZa) neural precursors differentiated. The cell populations are induced to differentiate from passage 3 of SVZ precursors (A-1) and passage 4 of SVZa precursors (A-2). Passage 4 of SVZ precursors were transfected with pEGFP-mDIx5 (A-3). B, Quantification and comparison of the data in A. One-way ANOVA confirmed that the differences between $A-1$ and $A-2, A-2$ and $A-3$ groups were significant $\left({ }^{*} P<0.01,{ }^{* *} P<0.001\right)$. NSE $=$ neuronspecific enolase. 
across brain areas, such as SVZa-RMS-OB, hippocampus, and subcortex. Islet1, on the other hand, is not expressed in these areas but is localized in the striatum. Interestingly, we observed that DIx 5 was negative but Er81 was positively expressed in the striatum area, again differing from the previous report (16). In addition, the expression of endogenous DIx5 mRNA in cultured SVZa neural precursors was gradually down-regulated and was completely lost by the fourth passage. Exogenous DIx 5 transfected into passage 4 precursors could stabilize the levels of DIx5 and promote the neuronal differentiation of cultured SVZa neural precursors. These data demonstrated that DIx5 plays an important role during neuronal differentiation. Further investigation is required to elucidate the underlying mechanism.

\section{The expression of DIx5 in SVZa-RMS-OB and SVZa neural precursors}

Wichterle et al. (17) studied the migration pathway of embryonic lateral ganglionic eminence (LGE) cells and found that LGE cells continuously migrate to the $O B$ and become the major source of $\mathrm{OB}$ interneurons. However, not all LGE cells migrate to the $\mathrm{OB}$; some cells migrate to the striatum and become striatal projection neurons. These results suggest that there are at least two populations of neural precursors in the LGE. Further studies by Stenman et al. (16) showed that only the cells in the dorsolateral LGE migrate to the $O B$ and differentiate into $O B$ interneurons. These cells have distinct molecular characteristics: they are positive for DIx and Er81 protein but negative for Islet1. Most of the cells in the ventral lateral LGE migrate toward the striatum and eventually differentiate into striatal interneurons and projection neurons, which are positive for DIx and Islet1 but negative for Er81. Because the SVZa neural precursors also directionally migrate to the $\mathrm{OB}$ and differentiate there, we hypothesized that SVZa neural precursors exhibit the same molecular features as LGE dorsolateral cells. Specifically, we hypothesized that they are also positive for DIx and Er81 and negative for Islet1.

In the present investigation, we studied the expression of DIx5 in SVZa-RMS-OB by IHC and found that DIx5 is widely expressed in a multitude of areas, including the SVZaRMS-OB, hippocampus, and subcortex. However, Er81 is also widely expressed, not only in these areas but also in the striatum. Hence, DIx5 and Er81 expression is high and overlaps in the SVZa-RMS-OB. On the other hand, Islet1 is not expressed in the SVZa-RMS-OB, but is expressed in the striatum. The expression of DIx5 in SVZa-RMS-OB suggests a close relationship between the DIx 5 gene and the process of migration and differentiation of $O B$ interneurons. It also confirms our hypothesis that SVZa precursors and LGE dorsolateral cells possess similar molecular features, i.e, they are positive for DIx and Er81 and negative for Islet1. Previous studies have shown that DIx 5 mRNA is highly expressed in forebrain subcortex GABA and dopamine neurons as well as in precursors of GABA neurons, but is low in differentiated neurons (11). Dlx5 appears to be preferentially expressed in neural precursors and stem cells. Therefore, the dorsolateral area of the LGE in embryonic stages may be similar to the postnatal SVZa structure. Furthermore, the SVZa neural precursors in neonates may originate from the dorsolateral area of the LGE.

Neural precursors isolated and cultured from the SVZa exhibited features of neural stem cells. Our data demonstrate that they continuously divide and proliferate, form suspended neurospheres in serum-free medium, show positive staining for the stem cell marker nestin (for a review, see Ref. 18), and can differentiate into neural cell types. These results suggest that, indeed, we successfully isolated neural precursors. Immunostaining confirmed that these precursors are positive for DIx5 and Er81 and negative for Islet1. We performed immunostaining studies on dorsolateral striatal neural precursors to compare the molecular characteristics of SVZa neural precursors and neural precursors from other brain areas. We found that these cells are Islet1 and Er81 positive but DIx5 negative. These data suggested that detecting DIx5, Er81, and Islet1 proteins in cultured SVZa neural precursors can facilitate exclusion of the precursors from other regions (at least the dorsolateral striatum). Islet1 and Er81 have been used as markers for striatal and OB interneurons, respectively (16), although their functions are still largely unknown. Our studies showed that DIx 5 is closely involved in the differentiation of OB interneurons from SVZa precursors and may play an important role in SVZa neural precursor differentiation.

\section{Effect of DIx5 on the neuronal differentiation of SVZa neural precursors}

After migration to the OB through the RMS, SVZa neural precursors terminally differentiated into OB interneurons. This process is regulated by multiple signaling events at both the transcriptional and post-transcriptional levels. Long et al. (14) showed that Dlx5 knock-out mice had few OB interneurons, suggesting that DIx5 plays a major role in the differentiation of $\mathrm{OB}$ interneurons and in the synaptogenesis of $\mathrm{OB}$ receptor neurons. The neural precursors isolated from the telencephalon of DIx5/- mice lost the ability to differentiate into neurons. Therefore, we speculated that DIx5 is related to the migration and neuronal differentiation of SVZ precursors. Dlx5/-- mice are neonatal lethal and therefore cannot be studied further. Hence, we studied the role of DIx5 in neuronal differentiation of SVZa neural precursors using recombinant plasmid and in vitro culture methods.

Green fluorescence protein is a small stable protein that does not interfere with the function of cells and shows no toxicity, even when overexpressed (19). It is a widely used marker for live fluorescent labeling. Here, we used the EGFP vector to construct the plasmid pEGFP-mDIx5. This permitted us to directly monitor mDlx 5 expression in SVZa neural precursors and to detect the cells expressing the target gene. Using this system, we confirmed the ex- 
pression of mDlx5-GFP fusion protein in transfected SVZa neural precursors by fluorescence microscopy. Because the molecular weight (MW) of GFP is approximately $27 \mathrm{kDa}$ and that of rat DIx5 is $31 \mathrm{kDa}$, the fusion protein should be approximately $58 \mathrm{kDa}$. Our Western blot data demonstrated that the MW of the fusion protein matches the expected MW (Figure 5B), thus further confirming that the recombinant plasmid can be used to study the role of DIX5 in the migration and differentiation of SVZ neural precursors.

The technique of live fluorescent labeling provided a very useful tool for analyzing the morphology of cells in the central nervous system. Here we studied the morphological changes in SVZa neural precursors following overexpression of GFP-mDIx5, demonstrating that the transfected cells differentiate into neuron-like cells. IHC staining for NSE confirmed that most of the DIx5-transfected cells differentiated into neurons. We further found that the expression of endogenous DIx5 decreased with increasing cell passage and became undetectable by RT-PCR in passage 4 cells. The Er81 expression level was unchanged during subsequent passages. Consistently, when the passage 4 precursors were induced to differentiate, less than $10 \%$ of cells successfully differentiated into neurons. This leads us to conclude that DIx5 levels may play an important role during the neuronal differentiation of SVZa neural precursors. This conclusion is further supported by the previous report that the Dlx genes encode homeodomain transcription factors related to Drosophila distal-less gene, and their mammalian homologs, Dlx1, Dlx2, DIx5, and DIx6,

\section{References}

1. Imayoshi I, Sakamoto M, Ohtsuka T, Kageyama R. Continuous neurogenesis in the adult brain. Dev Growth Differ 2009; 51: 379-386.

2. Quinones-Hinojosa A, Sanai N, Gonzalez-Perez O, GarciaVerdugo JM. The human brain subventricular zone: stem cells in this niche and its organization. Neurosurg Clin N Am 2007; 18: 15-20, vii.

3. Ayuso-Sacido A, Roy NS, Schwartz TH, Greenfield JP, Boockvar JA. Long-term expansion of adult human brain subventricular zone precursors. Neurosurgery 2008; 62: 223-229.

4. Ventura RE, Goldman JE. Dorsal radial glia generate olfactory bulb interneurons in the postnatal murine brain. $J$ Neurosci 2007; 27: 4297-4302.

5. Pencea V, Luskin MB. Prenatal development of the rodent rostral migratory stream. J Comp Neurol 2003; 463: 402418.

6. Lledo PM, Merkle FT, Alvarez-Buylla A. Origin and function of olfactory bulb interneuron diversity. Trends Neurosci 2008; 31: 392-400.

7. Alvarez-Buylla A. Mechanism of migration of olfactory bulb interneurons. Semin Cell Dev Biol 1997; 8: 207-213.

8. Haughey NJ, Liu D, Nath A, Borchard AC, Mattson MP. Disruption of neurogenesis in the subventricular zone of are expressed in the subcortical forebrain and are involved in the initiation of GABAergic neuron differentiation and neuronal migration $(10,11)$.

We have provided multiple lines of evidence demonstrating that Dlx5 plays an important role in the neuronal differentiation of SVZa neural precursors. First, DIx5 and Er81 are expressed in SVZa neural precursors; this expression pattern is one of the molecular features of SVZa precursors. In the in vitro cultured SVZa neural precursors, Dlx5 expression is gradually lost and can be regained by exogenous Dlx5 transfection, suggesting the existence of auto-regulation and external cue regulation. Taken together, these results demonstrate that DIx5 plays an important role during neuronal differentiation.

\section{Acknowledgments}

The authors thank Prof. Giovanni Levi (Co-Director of the Laboratory of Physiology, Museum National d'Histoire Naturelle, France) for his gift of pBluescriptBSK-mDIx5 plasmid. Sincere thanks are due to Ms. Wei Sun and Ms. Li-Ting Wang (senior experimental division and assistant laboratory technician, respectively, Central Laboratory, Third Military Medical University, Chongqing, China) for their assistance with the confocal laser scanning microscopy. The Chinese National Natural Science Foundation (\#30130110, \#30600638) and Chongqing Natural Science Foundation (\#2007BA5009, \#2008BB5032 and \#2007BB5025) financially supported this study. adult mice, and in human cortical neuronal precursor cells in culture, by amyloid beta-peptide: implications for the pathogenesis of Alzheimer's disease. Neuromolecular Med 2002; 1: 125-135.

9. Liu SY, Zhang ZY, Song YC, Qiu KJ, Zhang KC, An N, et al. SVZa neural stem cells differentiate into distinct lineages in response to BMP4. Exp Neurol 2004; 190: 109-121.

10. Perera M, Merlo GR, Verardo S, Paleari L, Corte G, Levi G. Defective neuronogenesis in the absence of Dlx5. Mol Cell Neurosci 2004; 25: 153-161.

11. Panganiban G, Rubenstein JL. Developmental functions of the Distal-less/DIx homeobox genes. Development 2002; 129: 4371-4386.

12. Merlo GR, Zerega B, Paleari L, Trombino S, Mantero S, Levi G. Multiple functions of Dlx genes. Int J Dev Biol 2000; 44: 619-626.

13. Levi G, Puche AC, Mantero S, Barbieri O, Trombino S, Paleari $\mathrm{L}$, et al. The DIx5 homeodomain gene is essential for olfactory development and connectivity in the mouse. Mol Cell Neurosci 2003; 22: 530-543.

14. Long JE, Garel S, Depew MJ, Tobet S, Rubenstein JL. DLX5 regulates development of peripheral and central components of the olfactory system. J Neurosci 2003; 23: 568-578. 
15. Luskin MB, Zigova T, Soteres BJ, Stewart RR. Neuronal progenitor cells derived from the anterior subventricular zone of the neonatal rat forebrain continue to proliferate in vitro and express a neuronal phenotype. Mol Cell Neurosci 1997; 8: 351-366.

16. Stenman J, Toresson $\mathrm{H}$, Campbell K. Identification of two distinct progenitor populations in the lateral ganglionic eminence: implications for striatal and olfactory bulb neurogenesis. J Neurosci 2003; 23: 167-174.

17. Wichterle H, Turnbull DH, Nery S, Fishell G, Alvarez-Buylla
A. In utero fate mapping reveals distinct migratory pathways and fates of neurons born in the mammalian basal forebrain. Development 2001; 128: 3759-3771.

18. Kempermann G, Jessberger S, Steiner B, Kronenberg G. Milestones of neuronal development in the adult hippocampus. Trends Neurosci 2004; 27: 447-452.

19. Moriyoshi K, Richards LJ, Akazawa C, O'Leary DD, Nakanishi S. Labeling neural cells using adenoviral gene transfer of membrane-targeted GFP. Neuron 1996; 16: 255-260. 